\title{
The psychophysical dimensions of similarity among random shapes ${ }^{1}$
}

HERBERT H, STENSON

BEHAVIOR RESEARCH LABORATORY, ANTIOCH COLLEGE

Six psychological dimensions were recovered from similarity judgments of 20 random forms using Kruskal's nonmetric scaling technique. These were related to 10 physical factors describing the forms by using canonical correlation analysis and rotations of the resulting vectors. Four factors accounted for $2 / 3$ of the variance of the intersection of the physical and psychological spaces for each of $11 \mathrm{Ss}$. The factors are described as Complexity, Curvature, Curvature Dispersion, and Straight-length Dispersion. The problem of the proper psychological distance metric is discussed, and individual differences among Ss are analyzed. The results lead to the conclusion that the four major factors found may be good representations of processes occurring in the perceptual system.

The judgment of similarity among members of a set of objects requires an observer to make simultaneous use of all the perceptual dimensions that he deems relevant. Thus, data on judged similarities contain information on the dimensions of perception. A variety of techniques for recovering the nature and the number of these perceptual dimensions from similarity data has been developed, beginning with the work of Richardson (1938) and the theorems of Young and Householder (1938). Torgerson (1958) reviews the scaling theory underlying the techniques in use up until the time of his book. Since Torgerson's book, important breakthroughs have been accomplished in the analysis of similarities by Shepard (1962) and Kruskal (1964).

The work of Kruskal2, to be described later, is of major concern in the present study. Kruskal's method is used here to recover the psychological dimensions underlying the judged similarities among members of a set of random shapes. These psychological dimensions are then related through the use of canonical correlation analysis to a set of physical dimensions that describe the same set of shapes. The result of this analysis is a physical description of the intersection of a set of psychological measures of these shapes with a set of physical measures of the same shapes.

The following sections on the general nature of the similarity scaling model, the non-metric scaling technique, and canonical correlation are designed to introduce the reader informally to concepts necessary for the understanding of this study. So as not to break the continuity between this intro- duction to the concepts and their use in the following sections, the discussion of literature pertinent to the substantive results of this study has been deferred to the Discussion section. There the results of relevant studies are stated and compared with the results of the present study.

\section{The Model}

The basic model on which this study is based is quite an old one. It is the same general model as that described by Torgerson (1958) for analyzing similarity data, and the same model forms the basis for the methods of Shepard (1962) and Kruskal (1964).

In this model the percept of a stimulus object is represented as a point located in a multidimensional Cartesian coordinate system, whose reference axes are (unknown) dimensions of perception. The points representing two very similar percepts are close to each other in this psychological space, and very dissimilar percepts are represented by points that are far apart. The interpoint distances, which represent similarities, have usually been treated as Euclidean distances: That is, the distance between any two points is equal to the squared differences of their projections on each reference axis of the space summed over all axes. A notable exception to this practice is. Attneave's "city block" distance model for form perception (Attneave, 1950). In this model the "distance" between any two points in the psychological space is the sum of the absolute differences of their projections on each axis of the space.

The data collected in the study of similarities are usually ordinal in nature, e.g., we ask Ss whether one pair of stimuli is more similar than another pair, or to choose the most similar pair in each possible tetrad of stimuli, or to rate each possible pair of stimuli on a category scale of similarity, and so on. The problem then is to transform these ordinal data to ratio-scale data that are taken to be the interpoint distances in the model described above. Various scaling techniques have been devised to accomplish this transformation, most of which are variations and adaptations of Thurstone's laws of comparative and categorical judgment (see Torgerson, 1958, p. 261).

Assuming a Euclidean distance model, it is a fairly simple matter to construct a conceptual space from which a set of interpoint distances could have come, given ratio-scale data for these distances. 
However, there has been dissatisfaction with the assumptions required to scale ordinal similarity data in such a way as to achieve a proper ratio scale. It is in this area that the breakthrough has come. The multidimensional scaling methods developed by Shepard (1962) and refined by Kruskal (1964) enable one to construct the space from which the ordinal data must have come without first having to perform a unidimensional scaling of interpoint distances. Thus, these methods are referred to as nonmetric.

\section{Nonmetric Mulfidimensional Scaling}

Bennett (1956) and Bennett and Hays (1960) demonstrated that in a space with fewer dimensions than points, the rank order of interpoint distances constrains the possible locations of the points. If the number of dimensions is held constant and the number of points increased, the constraint imposed by ranking on the location of each point increases. In the limit, the location of each of an infinite number of points in a finite-dimensional space is completely determined by the rank order of the interpoint distances if one of the interpoint distances is already specified.

Shepard (1962) examined this same phenomenon and demonstrated that the constraints imposed by the rank.ordering of distances increase very rapidly as the number of points increases in a space of fixed dimensionality. For example, Shepard obtained an extremely good reconstruction of the configuration of only 15 points in a plane by using the constraints implied by the $\left(\frac{15}{2}\right)$ interpoint distance rankings.

Kruskal (1964) studied the same problem, refined Shepard's method, and added a "goodness-of-fit" measure to it. To attain an intuitive grasp of Kruskal's method, suppose that we have data consisting of a similarity ranking for each of $\left(\frac{\mathrm{n}}{2}\right)$ pairs of $n$ stimulus objects. The rankings are the input to the Kruskal computer program, MDSCAL (multidimensional scaling program). This program first generates an arbitrary configuration of $n$ points in an m-dimensional space, where $m$ is specified by the experimenter. It then computes the $\left(\frac{\mathrm{n}}{2}\right)$ interpoint distances among these arbitrarily numbered points and compares them with the rankings of the corresponding stimulus pairs as given by the Ss. The degree to which the relationship between distances and rankings is monotonic is expressed by a goodness-of-fit measure called "Stress." The MDSCAL program then moves the $n$ points slightly in the m-space in such a way as to improve the fit (decrease Stress). This process is repeated until a minimum Stress is achieved. The same procedure may be followed for various values of $\mathrm{m}$, and the minimum Stress is obtained for each value of $m$. The smallest dimensionality that produces a satisfactory fit, as indicated by Stress, is then chosen as the best representation of the dimensionality of the conceptual space from which the similarity rankings must have come. The coordinates of each of the $n$ points in this space are then obtained using an arbitrary unit of measure.

A parameter is provided in the MDSCAL program to control the distance metric used in the program. One may use Euclidean distances, city-block distances, or any other of a class of distance metrics referred to as Minkowski r-metrics (see Kruskal, 1964). Assuming that Euclidean distance is used, one obtains the coordinates of $n$ points in a bestfitting $m$-space with an arbitrarily oriented set of reference axes. If a non-Euclidean distance metric is used, one obtains the coordinates of $n$ points in a best-fitting m-space the locations of whose reference axes are fixed by the interpoint distances in the configuration of points. Thus, the reference axes can be rotated without affecting the interpoint distances only if the Euclidean metric is used.

In the present study Euclidean distances are used in the analysis of the psychological data, and the physical space defining the shape stimuli is also Euclidean. This approach allows a choice of reference systems in both the physical and psychological spaces, a situation in which canonical correlation analysis can provide the quantitative basis for the establishment of a multidimensional psychophysical relationship.

\section{Canonical Analysis}

Whenever two different sets of measurements are available on the same set of objects, the interrelations of the measurement sets can be studied by canonical-correlation techniques. Hotelling $(1935,1936)$ developed canonical correlation as a method of obtaining the maximum correlation between a single pair of linear combinations of two sets of variables, one member of the pair from one set of variables and one from the other set. The technique may be extended to obtain correlations between successive pairs of linear combinations within the two measurement sets. Each successive pair is determined in such a way as to maximize the correlation between the members of that pair, subject to the restriction that they be independent of both members of all previous pairs of linear combinations. The productmoment correlation coefficients between the successive pairs of linear combinations of measures are called the canonical correlation coefficients, the linear combinations themselves are called canonical vectors, and the weights applied to the original measures in order to obtain the canonical vectors are called the canonical weights.

Alternatively, one may consider canonical correlation to be a method of measuring the amount of variance that two sets of normalized measurements of the same objects have in common. That is, the relative size of the intersection of two vector spaces 
is measured. In the present paper the size of the intersection between a set of normalized physical measures and a set of normalized psychological measures on the same set of random shapes is found for each $\mathrm{S}$. The intersection is then interpreted as the physical basis for the perceptual dimensions that generate the judgments of similarity among the shapes. The psychological measures are derived from similarity judgments analyzed by the Kruskal MDSCAL program as described earlier, and the physical measures are the scores of each of the random shapes on a set of 10 physical factors describing these shapes as given by Stenson (1966).

\section{Subjects}

\section{METHOD}

The Ss were 11 undergraduates at Antioch College who were paid to participate in the experiment.

\section{Stimuli}

Twenty outline shapes drawn according to Method 4 of Attneave and Arnoult (1956) were used as stimuli. These shapes are exhibited by Stenson (1966). Each of the 190 possible pairs of these stimuli was printed on a $4 \quad x \quad 8$ in. card. Within each pair the member of the pair that was to appear on the righthand half of the card was randomly determined.

\section{Procedure}

Each $\mathrm{S}$ was run individually for $1 \mathrm{~h}$ on each of five successive days. On his first day each $S$ was shown a display of all 20 shapes and told that his task would be to judge the similarity between the members of each possible pair of shapes. He was then shown to a table on which was a pack of the 190 cards containing the stimulus pairs. He was asked to leaf through the pack, looking at each pair of shapes, and mentally note the degree of similarity between the members of each pair. The pack of cards was then shuffled and the experimental trials began.

The $S$ was instructed to sort the pack of cards into two piles, placing cards with the more similar pairs of shapes in a pile to his left and those with the more dissimilar pairs in a pile to his right. $\mathrm{He}$ was told to set his criterion for similarity in such a way that the two piles would not differ greatly in size. Two small signs reading "More Similar" and "More Dissimilar" were placed on the table to the S's left and right, respectively, to continually remind him of the pile designations. Each of the two resulting piles of cards was then shuffled by the $\mathrm{E}$, and the piles were moved a short distance apart on the table. The $S$ was then asked to sort the lefthand pile and then the righthand pile each into two piles, a similar and a dissimilar pile. The four resulting piles were then shuffled by the $E$, and the $S$ was asked to sort each into two more piles beginning with the leftmost of the four piles and working toward the right. This same sorting procedure was followed once more on the resulting eight piles. At the end of the fourth sort the $S$ had produced 16 piles of cards ranging from cards with the most similar pairs at the extreme left end of the table to cards with the most dissimilar pairs at the extreme right. He was then asked to look through each pile of cards, beginning with the leftmost pile and working right, and was told that if he found a card that should not be in the particular pile he was looking at, he was free to move it to any other pile. This same procedure (beginning with the sorting) was followed in each of the four following sessions. The pile number (1-16) for each card was recorded after each session. This sorting method was devised by Silver, Landes, and Messick (1966) for use in a study similar to the present one.

\section{Analysis of Similarities}

The data for each $\mathrm{S}$ was analyzed separately. First the average pile number for each pair of shapes over the five sessions was computed. The 16 piles and five sessions made possible a 76 point scale $(5 \times 16-4)$ of average pile numbers (APN) over the five days. The APN data were the input for the MDSCAL program. The program, as described earlier, relates computer-generated, interpoint distances to the rankings received from the Ss. If some of the rankings are duplicated, that is, the stimuli are only partially ordered, as is the case with the APN data in the present study, two program options are available. The Primary Method (see Kruskal, 1964) allows a single ranking to correspond to more than one interpoint distance in the psychological space, subject to the restriction that the distances and rankings be monotonically related to as great a degree as possible. In the Secondary Method if two pairs of stimuli are ranked the same, the program requires that the interpoint distances for these pairs be made equal. The Primary Method was used for the present data analysis.

The number of dimensions in which the APN data were analyzed was varied from 10 dimensions to one dimension in steps of one. Thus, Stress (the goodness-of-fit measure) was obtained for each of 10 dimensionalities for each $\mathrm{S}$. Stress is an index number that can vary from zero to one, with zero Stress indicating a perfect fit. Kruskal (1964) states that his "...experience with experimental and synthetic data suggests the following verbal evaluation" of the Stress measure: Stress of $20 \%$ or more indicates a poor fit, $10 \%$ indicates a fair fit, $5 \%$ a good fit, and $2.5 \%$ or less an excellent fit. Zero Stress indicates a perfect monotone relationship between similarities and distances, 
The Stress for each dimensionality used was examined, and the appropriate dimensionality was decided upon for each $\mathrm{S}$ for further analysis.

\section{Physical Measures of Stimuli}

The physical data to which the psychological data are related are the factor scores of each of the random shapes on 10 simple-structure, orthogonal factors found by Stenson (1966) to account for $94 \%$ of the total variance in 24 physical measures describing the shapes. Table 1 provides a name for each of these factors and shows the physical measures that had the highest loadings on them. A brief description of each of these factors follows.

The Curvature factor may be thought of as the proportion of the perimeter of a shape that is composed of ares, while the Curvature Dispersion factor is a measure of the diversity of arc lengths in the perimeter. The Orientation factor may be simply described as the angle that a line drawn through the maximum extent of the shape makes with the horizontal. Complexity is a factor defined by the variables found by Attneave (1957) and Arnoult (1960) to be highly related to the judged complexity of random shapes. Stenson (1966) found that complexity ratings were highly related $(r=.90)$ to a single factor described by these same variables. Straight-length Dispersion measures the diversity in the lengths of straight segments in the perimeter of a shape. The general meaning of Height-Width Ratio is obvious, but it should be noted that this factor does not simply measure the ratio of maximum vertical

Table 1. Ten Factors Describing $94 \%$ of the Variance of Physical Measures Made on 20 Forms, and the Physical Measures that De fine Each Factor

\begin{tabular}{llr} 
Foctor & \multicolumn{1}{c}{ Defining Measures } & Loading \\
\hline A. Curvature & $\begin{array}{l}\text { Mean Straight Length } \\
\text { \% Arcs }\end{array}$ & -.93 \\
& \% Straight Perimeter & .90 \\
B. Curvature Dispersion & $\begin{array}{l}\text { Variance Arc Length } \\
\text { Mean Arc Length }\end{array}$ & .96 \\
& Skewness Arc Length & .83 \\
C. Orientation & Elongation Index & -.78 \\
& Orientation Angle & .81 \\
D. Complexity & Perimeter (P) & .85 \\
& Number of Turns in P & .83 \\
& P2/Area & .80 \\
E. Straight-Length & Variance Internal Angles & .70 \\
Dispersion & Skewness Straight Length & -.72 \\
F. Height-Width Ratio & Variance Straight Length & -.69 \\
& Variance on X-axis & -.91 \\
G. Topheaviness & Variance Index & .88 \\
H. Angular Concavity & \% Convex Angles & -.93 \\
I. Curved Concavity & \% Convex Arcs & -.86 \\
J. Angular Asymmetry & Skewness Internal Angles & -.92 \\
\hline
\end{tabular}

extent to maximum horizontal extent. Rather, the shape is viewed as a bivariate statistical distribution of unit density within the form and zero elsewhere. The factor is best defined by the Variance Index, which is the difference between the variances along the $x$ and $y$ axes of this distribution divided by the sum of these variances. The skewness (third central moment) along the vertical axis when the shape is treated as a bivariate density function defines the Topheaviness factor. The meanings of Angular Concavity and Curved Concavity are given respectively by the proportion of the angles in the perimeter that are concave when viewed from the interior of the shape and the proportion of the arcs in the perimeter that are concave when viewed from the interior of the shape. The last factor, Angular Asymmetry, is defined by the skewness (third central moment) of the distribution of angles contained in the perimeter.

It should be noted that when 12 factors rather than 10 factors were retained and rotated for this set of shapes, about $97 \%$ of the total variance of the physical measures was accounted for, with the two additional factors described by Area and by Skewness along the horizontal axis of the shape. The Iatter is the horizontal counterpart of Factor G, Topheaviness. These factors contributed so little to the total variance of this particular set of shapes that they were omitted from the analysis. However, it is reasonable on logical grounds to expect them to be important descriptors for other sets of shapes where the area and the degree of skewness along the horizontal axis contribute more variance.

\section{Canonical Analysis}

The next step of the analysis of the similarities among shapes was to relate the psychological space for each $S$ as represented by the results of the MDSCAL analysis to the physical space represented by the physical factors just described. Canonical correlation analysis was used for this purpose. Note that the physical variables (factors) that entered into the canonical analysis were independent and normalized at the outset of the canonical analysis. (The physical factors will be referred to as "physical variables" in what follows in order to avoid ambiguities.) This characteristic of the physical variables forces independence among the sets of canonical weights for the physical variables, and makes the following type of analysis possible for each $\mathbf{S}$.

The members of each set of canonical weights for the physical variables were multiplied by the canonical correlation coefficient corresponding to the given set of weights. This process produced a matrix very similar to a principal axes factor matrix because of the independence among sets of canonical weights for the physical variables. In this matrix the "loadings" of each physical variable on 
a given psychological "factor" are a set of weights showing the relationship of the physical variables to one dimension of the psychophysical intersection. These psychological factors were then rotated to simple-structure using the Varimax criterion. The resulting matrix was interpreted as a physical basis of psychological judgments of similarity. The mathematics underlying this analysis are given in the Mathematical Appendix.

\section{MDSCAL Analysis}

\section{RESULTS}

Table 2 shows Stress as a function of dimensionality for each $\mathrm{S}$. The MDSCAL program was set to discontinue iterations for the dimensionality on which it was working if stress reached $5 \%$ or less, or if a minimum Stress greater than 5\% was reached. Stress is shown for only 1 through 7 dimensions in Table 2 because Stress had reached $5 \%$ for all Ss for dimensionalities of 8,9 , and 10 .

For no $S$ is there an abrupt change in the downward progression of Stress as dimensionality is increased. Such a change would help to determine the proper dimensionality to use for an $S$ (see Kruskal, 1964). Therefore, the 5\% Stress level was used as a criterion to determine the proper dimensionality. Table 2 shows that with five dimensions Stress for all Ss is greater than $5 \%$, although S No. 5 is close to $5 \%$. For six dimensions the Stress for all Ss is at or very near the $5 \%$ criterion, and for seven dimensions the criterion is met for all Ss. Each increase in dimensionality decreases the constraints on the locations of points in the conceptual space. Therefore, the results for six dimensions were used for further analysis, even though the 5\% criterion is not rigidly met for all Ss. The Stress for all Ss is lower than $6.8 \%$ when six dimensions are used. The Stress for $S$ No. 5 is sufficiently close to $5 \%$ at five cilmensions to warrant using five rather than six dimensions for him, but for the sake of uniformity of analysis, six dimensions

Table 2. MDSCAL Stress Values for Each of 7 Dimensionalities for Each of 11 Ss

\begin{tabular}{cccccccc}
$\mathrm{S}$ & 1 & 2 & 3 & 4 & 5 & 6 & 7 \\
\hline 1 & .324 & .229 & .144 & .097 & .075 & .056 & .050 \\
2 & .347 & .184 & .135 & .086 & .064 & .050 & .050 \\
3 & .413 & .221 & .148 & .103 & .068 & .054 & .050 \\
4 & .376 & .254 & .160 & .111 & .084 & .068 & .050 \\
5 & .229 & .142 & .097 & .073 & .052 & .050 & .049 \\
6 & .340 & .174 & .108 & .080 & .058 & .050 & .050 \\
7 & .340 & .213 & .166 & .111 & .075 & .057 & .050 \\
8 & .360 & .198 & .113 & .074 & .062 & .050 & .049 \\
9 & .340 & .152 & .109 & .084 & .064 & .053 & .049 \\
10 & .244 & .186 & .138 & .083 & .065 & .052 & .050 \\
11 & .410 & .225 & .149 & .101 & .075 & .059 & .050 \\
Median & .340 & .198 & .138 & .086 & .065 & .053 & .050 \\
\hline
\end{tabular}

were used for all Ss. The goodness-of-fit may be said to be good in six dimensions for all Ss, using the verbal criteria stated earlier.

\section{Canonical Analysis}

The 6-dimensional MDSCAL data and the 10dimensional physical data were subjected to a canonical correlation analysis for each $\mathrm{S}$ independently, resulting in the canonical correlation coefficients shown in Table 3 . No test of statistical significance was done on the canonical coefficients for reasons to be discussed later.

The final column of Table 3 shows the percentage of the total variance in the psychological space that can be accounted for by the six canonical vectors, in the physical space. These percentages are obtained by squaring and summing the canonical coefficients for an $S$ and then dividing this sum by six, the total variance of the normalized psychological space.

For each $\mathrm{S}$ each of the six sets of canonical weights for the physical variables was multiplied by its corresponding canonical coefficlent. This produced a matrix for each $S$ in which the rows corresponded to the 10 physical variables named in Table 1 , and the columns were six unnamed psychological axes. The six columns of each of the 11 resulting matrices (one for each $S$ ) may be viewed as six independent principal axis factors from a factor analysis of the psychophysical intersection. The squared canonical coefficients are the variances along these factors. A rotation of each matrix, using the Varimax criterion, produced a 10 by 6 matrix with simple-structure for each $\mathrm{S}$.

Table 4 is an example of the results of this treatment of the data for a single $S$. The matrix shown is a simple-structure description of the intersection of the physical and psychological spaces for S No. 7. The last row of Table 4 shows the percentage of the intersection that is accounted for by each of the six factors. These percentages are analogous to "the percentages of common variance accounted

Table 3. Canonical Correlation Coefficients for Psychological and Physical Canonical Vector Pairs, and Percentage of Total Variance Accounted for by these Correlations for Each of 11 Ss

\begin{tabular}{cccccccc} 
& \multicolumn{9}{c}{ Canonical Vector Pair } & \% of Total \\
$\mathrm{S}$ & 1 & 2 & 3 & 4 & 5 & 6 & Variance \\
\hline 1 & .98 & .91 & .85 & .72 & .63 & .46 & .61 \\
2 & .99 & .98 & .92 & .88 & .63 & .43 & .70 \\
3 & .99 & .98 & .89 & .84 & .69 & .35 & .67 \\
4 & .98 & .96 & .93 & .85 & .57 & .38 & .65 \\
5 & 1.00 & .98 & .92 & .84 & .78 & .36 & .71 \\
6 & .99 & .92 & .91 & .71 & .60 & .47 & .62 \\
7 & .98 & .94 & .86 & .81 & .69 & .63 & .68 \\
8 & .99 & .95 & .86 & .81 & .59 & .49 & .65 \\
9 & .98 & .93 & .75 & .71 & .62 & .57 & .60 \\
10 & .98 & .95 & .88 & .66 & .58 & .41 & .60 \\
11 & .99 & .97 & .88 & .82 & .77 & .57 & .72 \\
Median & .99 & .96 & .88 & .84 & .63 & .47 & .65 \\
\hline
\end{tabular}


Table 4. A Simple-Structure Representation of the

Psychophysical Intersection for $\mathbf{S}$ No. 7

\begin{tabular}{lccrrrr}
$\begin{array}{l}\text { Physical } \\
\text { Variable }\end{array}$ & \multicolumn{7}{c}{ Psychological Axes } \\
\hline A. & -.15 & .75 & .04 & .03 & .05 & -.10 \\
B. & .00 & .05 & .70 & -.12 & .09 & .05 \\
C. & .00 & -.04 & .08 & .04 & .68 & .02 \\
D. & .82 & -.16 & -.01 & .03 & -.02 & -.01 \\
E. & .01 & -.02 & -.11 & .60 & .04 & .05 \\
F. & -.17 & -.23 & -.19 & .11 & .19 & .13 \\
G. & -.02 & -.09 & .07 & .09 & .05 & .63 \\
H. & -.23 & -.21 & .26 & .19 & -.17 & .05 \\
I. & -.05 & -.14 & .27 & .33 & -.38 & -.36 \\
J. & .04 & -.26 & .05 & .15 & .12 & -.21 \\
\% of & & & & & & \\
Intersection & 19 & 19 & 17 & 14 & 17 & 15 \\
\hline
\end{tabular}

for" in the usual factor analysis. For this $\mathrm{S}$ the intersection of the two spaces contains $68 \%$ of the total variance in the psychological space as shown in Table 3. That is, the common variance which is apportioned in Table 4 is $68 \%$ of the total variance, to use the parlance of factor analysis. A column of any one of the 11 simple-structure matrices (one for each S) obtained by this method will be called an Intersection Factor (IFAC).

Tables 5, 6, 7, and 8 summarize some of the results in the 11 IFAC matrices, of which Table 4 is an example. Each of these tables shows the physical structure of a particular IFAC for each $S$ who had an IFAC appropriate for the table in question. The tables were constructed as follows. A single physical variable was chosen for consideration, and the corresponding row of an S's IFAC matrix was scanned for the highest (absolute) loading. When this maximum loading was found, the column (IFAC) containing it was scanned for higher loadings. If no higher loading was found, that IFAC was entered into a table labelled with the name of the physical variable under consideration. (Reflections of factors were used when appropriate, so that all maximum loadings were positive.) This proce- dure was followed with each physical variable. For example, Column 7 in Table 5 contains a loading of .82 for physical variable $D$, Complexity. Table 4 shows that .82 is the highest loading that variable $D$ has on any IFAC for S No. 7 and also is the highest loading on the IFAC in question. Thus, Column 1 from Table 4 was entered in Table 5 for S No. 7 . If, for a given $S$, the highest loading for a particular physical variable was not also the highest loading on the corresponding IFAC, no entry was made for that $S$ in the table bearing the name of the physical variable in question. For example, Column 2 of Table 6 is filled with Xs. This means that for S No. 2 there was no IFAC for which physical variable $A$, Curvature, had the highest loading.

Thus, Table 5 shows that all 11 Ss had an IFAC best described by physical variable $D$, Complexity. Table 6 shows that each of 10 Ss had an IFAC best described by physical variable A, Curvature. Table 7 shows that each of $10 \mathrm{Ss}$ had an IFAC best described by physical variable $B$, Curvature Dispersion. And Table 8 shows that all 11 Ss had an IFAC best described by physical variable E, Straight-length Dispersion. The last column of each of these tables shows the median factor loadings for the Ss entering into the table. The last row of each table shows for each $S$ the percentage of the psychophysical intersection that is accounted for by the IFAC in question and the median of these percentages.

As a summary of the data up to this point, it may be noted that the 10 physical variables whose names are listed in Table 1 account for about $2 / 3$ of the variance in the psychological space on the average (see Table 3), and that for most Ss, four of these physical variables account for about $3 / 4$ of the variance in this psychophysical intersection (see Tables $5,6,7$, and 8). Thus these four physical variables, Complexity, Curvature, Curvature Dispersion, and Straight-length Dispersion, account on the average for about $1 / 2$ of the total variance $(3 / 4$ of $2 / 3)$ in the psychological space.

Table 5. Complexity Intersection Factor for Each of 11 Ss, Showing Factor Loadings of Physical Variables, Percentage of Intersection Variance Accounted for $(\%)$, and Medians (Md) over Ss. (Decimal points omitted)

\begin{tabular}{|c|c|c|c|c|c|c|c|c|c|c|c|c|}
\hline $\begin{array}{l}\text { Physical } \\
\text { Factor }\end{array}$ & 1. & 2. & 3. & 4. & 5. & $\begin{array}{c}\text { bject } \\
6 .\end{array}$ & 7. & 8. & 9. & 10. & 11. & $\mathrm{Md}$ \\
\hline A. & 02 & -39 & -19 & 03 & 00 & -09 & -15 & -35 & -08 & -17 & -07 & -08 \\
\hline B. & 04 & -05 & -08 & -09 & 02 & -07 & 00 & -08 & -09 & 13 & -11 & -07 \\
\hline C. & -06 & 01 & 01 & 12 & -03 & 06 & -00 & 04 & -04 & -06 & 07 & 01 \\
\hline D.* & 74 & 81 & 75 & 88 & 88 & 86 & 82 & 79 & 83 & 90 & 86 & 83 \\
\hline E. & -03 & 13 & -13 & 01 & 06 & -08 & 01 & 03 & -05 & 02 & -11 & 01 \\
\hline F. & -03 & -09 & -25 & -16 & -03 & -08 & -17 & -07 & -04 & -05 & -15 & -07 \\
\hline G. & -04 & -04 & 01 & -08 & -14 & 01 & -02 & 01 & -12 & -07 & -09 & -04 \\
\hline $\mathrm{H}$. & -47 & -03 & -24 & -07 & -37 & -10 & -23 & -17 & -02 & -08 & -13 & -13 \\
\hline 1. & -03 & 02 & 08 & -09 & -06 & 07 & -05 & 09 & -01 & -00 & 02 & 00 \\
\hline$\ddot{j}$. & -14 & 00 & 13 & 06 & 05 & -07 & 04 & 04 & 04 & 04 & 02 & 04 \\
\hline$\%$ & 22 & 20 & 19 & 22 & 22 & 21 & 19 & 21 & 20 & 24 & 19 & 21 \\
\hline
\end{tabular}

* Criterion variable used to compile table. 
Table 6. Curvature Intersection Factor for Each of $10 \mathrm{Ss}$, Showing Factor Loadings of Physical Variables, Percentage of Intersection Variance Accounted for (\%), and Medians (Md) over Ss. (Decimal points omitted)

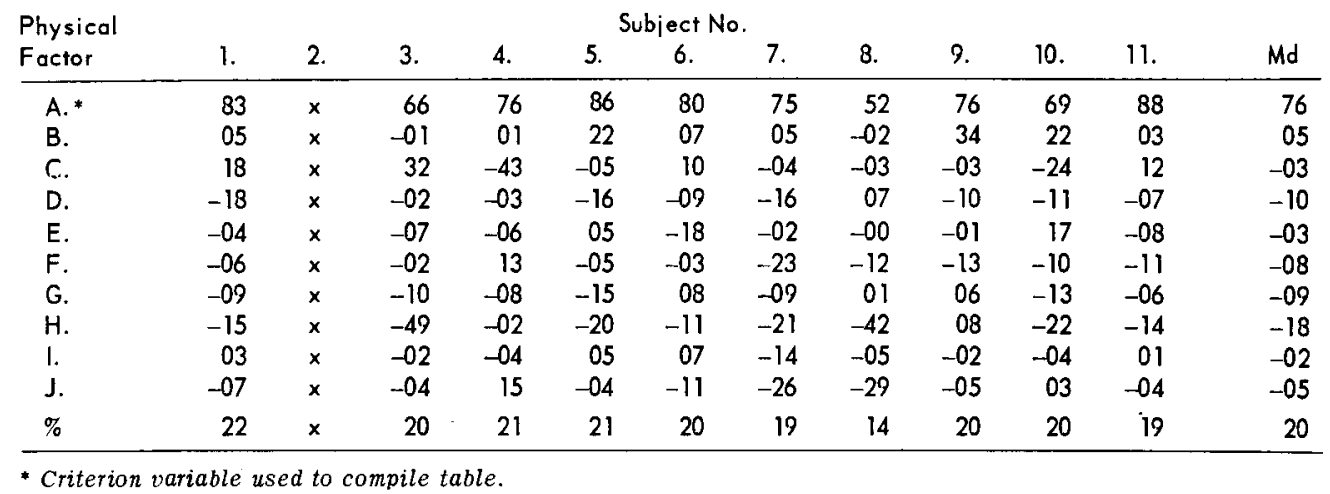

Tables similar to Tables 5-8 were constructed for each of the remaining six physical variables. However, only summary data from these tables are given here because the Ss were quite heterogeneous with regard to the structure of the remaining IFACs, and because the percentages of the variance accounted for by these remaining IFACs were, in most cases, substantially lower than for the four types of IFAC presented in Tables 5-8. Table 9 summarizes these remaining IFACs by presenting the median factor loadings from each table and the median percentages of variance accounted for. Thus, each column of Table 9 is the analogue of the last column (medians) of each of the preceding IFAC tables, Tables 5-8. The last row of Table 9 shows the number of Ss who had IFACs that qualified for inclusion in the table from which the medians in a given column in Table 9 were computed. The labels on the columns of Table 9 are the names of the tables from which the medians were computed. These are the names of the physical variables that were under consideration when compiling the tables on which Table 9 is based. Note that Table 9 shows that seven of the 11 Ss had an IFAC that is best described as Topheaviness, but that only three or four Ss' data contained any one of the remaining five types of IFAC.
There were, of course, 10 types of IFAC possible because 10 physical variables entered into the analysis, but there were only six psychological dimensions to account for. Therefore, only six of the 10 possible types of IFAC were possible for a given S. Table 10 shows the pattern of types of IFAC found for each $S$ by giving the percentage of the variance of each $S$ 's intersection that was accounted for by each of his six IFACs.

\section{DISCussion}

The major finding of this research is that four physical variables account for the majority of the variance in a conceptual psychological space whose dimensions are judgment axes of figural similarity. This finding is based on a chain of analyses, certain aspects of which require discussion.

First, the interpretation of the results relies on the acceptance of the model of conceptual space described earlier in an introductory section. While there seems to be general agreement among psychologists on the general nature of this model with regard to the judgment of similarity, there has been discussion of some critical features of the model and of some procedural matters related to its use. A critical feature that has received much attention

Table 7. Curvature Dispersion Intersection Factor for Each of 10 Ss, Showing Factor Loadings of Physical Variables, Percentage of Intersection Variance Accounted for (\%), and Medians (Md) over Ss. (Decimal points omitted)

\begin{tabular}{|c|c|c|c|c|c|c|c|c|c|c|c|c|}
\hline \multirow{2}{*}{$\begin{array}{l}\text { Physical } \\
\text { Factor }\end{array}$} & \multicolumn{12}{|c|}{ Subject No. } \\
\hline & 1. & 2. & 3. & 4. & 5. & 6. & 7. & 8. & 9. & 10. & 11. & Md \\
\hline A. & 07 & 17 & 12 & 17 & 03 & 08 & 04 & 25 & $x$ & -04 & 01 & 08 \\
\hline B.* & 69 & 80 & 72 & 66 & 57 & 58 & 70 & 63 & $x$ & 53 & 48 & 65 \\
\hline C. & 22 & 01 & 15 & 14 & -04 & 01 & 08 & 06 & $x$ & 09 & -26 & 07 \\
\hline D. & -05 & -02 & -07 & -04 & 02 & 06 & -01 & -07 & $x$ & -03 & -02 & -02 \\
\hline E. & -07 & 22 & -06 & 03 & 03 & -13 & -11 & 05 & $x$ & -06 & 03 & -01 \\
\hline $\mathrm{F}$. & 04 & -10 & -29 & -20 & -14 & -46 & -19 & 04 & $x$ & -01 & -46 & -16 \\
\hline G. & 13 & 23 & 07 & 14 & 32 & -04 & 07 & 16 & $x$ & 52 & 15 & 15 \\
\hline H. & -05 & -06 & 35 & 05 & 02 & -10 & 26 & 01 & $x$ & 13 & 46 & 04 \\
\hline 1. & -02 & -10 & -04 & -25 & -57 & -04 & 27 & -07 & $x$ & 05 & 02 & -04 \\
\hline J. & -05 & -05 & -03 & -07 & -07 & 01 & 05 & 02 & $x$ & 00 & 00 & -01 \\
\hline$\%$ & 15 & 19 & 19 & 16 & 18 & 16 & 17 & 13 & $x$ & 17 & 17 & 17 \\
\hline
\end{tabular}

* Criterion variable used to compile table. 
Table 8. Straight Length Dispersion Intersection Factor for Each of 11 \$s, Showing Factor Loadings of Physical Variables, Percentage of Intersection Variance Accounted for (\%), and Medians (Md) over Ss.

(Decimal points omitted)

\begin{tabular}{|c|c|c|c|c|c|c|c|c|c|c|c|c|}
\hline \multirow{2}{*}{$\begin{array}{l}\text { Physical } \\
\text { Factor }\end{array}$} & \multicolumn{12}{|c|}{ Subject No. } \\
\hline & 1. & 2. & 3. & 4. & 5. & 6. & 7. & 8. & 9. & 10. & 11. & Md \\
\hline A. & -08 & 11 & -15 & 07 & 04 & 05 & 03 & -15 & -01 & 05 & -07 & 03 \\
\hline B. & -09 & 03 & -05 & 08 & 14 & 36 & -12 & 06 & -19 & -03 & -15 & -03 \\
\hline C. & 08 & 47 & 37 & 27 & 11 & 34 & 04 & 13 & 05 & 32 & 16 & 16 \\
\hline D. & -06 & 10 & 05 & 02 & 14 & 04 & 03 & 01 & -06 & -03 & -06 & 02 \\
\hline E.* & 61 & 56 & 59 & 80 & 73 & 67 & 60 & 75 & 57 & 52 & 74 & 61 \\
\hline $\mathrm{F}$. & 17 & -04 & 24 & 24 & 10 & 16 & 11 & 29 & 20 & 17 & -03 & 17 \\
\hline G. & -09 & -07 & -01 & 02 & 18 & 02 & 09 & 08 & -02 & 05 & -13 & 02 \\
\hline $\mathrm{H}$. & 00 & 06 & 07 & 03 & 05 & 03 & 19 & 06 & -26 & 06 & 15 & 06 \\
\hline 1. & 13 & 24 & 04 & 19 & 21 & 11 & 33 & 13 & 23 & 18 & 40 & 19 \\
\hline J. & 17 & -07 & 34 & 16 & 10 & -09 & 15 & -03 & 01 & 02 & 02 & 02 \\
\hline$\%$ & 13 & 15 & 17 & 22 & 16 & 20 & 14 & 19 & 15 & 13 & 19 & 16 \\
\hline
\end{tabular}

* Criterion variable used to compile table.

is the nature of the proper distance metric to be used to describe the interpoint distances. The issue was first raised by Attneave (1950), who contended on the basis of his data that a "city-block" metric was appropriate for the model describing the judged similarities among a set of forms. The problem of distance metric with regard to the present study is discussed below.

Next, the statistical aspects of the data are discussed, after which a procedural matter relating to the effects of individual differences on the model is discussed. Consideration of the latter was prompted by an important paper by Tucker and Messick (1963), and by a study by Silver, Landes, and Messick (1966). The decision to analyze each S'S data separately in the present study reflects this consideration. Finally, the substantive results of this study, the psychophysical dimensions of figural similarity, are discussed, after which some conclusions are stated.

\section{Distance Metrics}

One of the assumptions underlying the present study is that a Euclidean distance metric is appropriate for the conceptual space in which the percepts of the stimuli are represented. Several aspects of the results tend to support this assumption. First, the four physical variables found to describe four major dimensions of the intersection of the psychological and physical spaces are plausible from an interpretive point of view. That is, the four factors in Tables 5-8 are verbally interpretable without involving highly abstract mathematical concepts. Second, the results are consistent with those from a study by Stilson (1966) where the task set for the S was not the same as in the present study, a different class of shapes was used, and the method of analysis was different from that used here. A comparison of Stilson's results with those of this study is made later. Third, there was a high degree of consistency between Ss in the present study with regard to the major physical variables correlated with their similarity judgments. Two of the four major physical variables in Tables 5-8 were important for all of the $11 \mathrm{Ss}$.

However, consistency and meaningfulness of results, using a Euclidean metric, do not imply that an even better fit and even more consistency could not be achieved by assuming a non-Euclidean metric. Kruskal's scaling program specifically provides a

Table 9. Median Factor Loadings for 6 Least Important Intersection Factors (IFAC) Showing Median Percentage of Intersection Accounted for $(\%)$, and Number of Ss (N) for Each IF AC (Decimal points omitted)

\begin{tabular}{lccccrr}
$\begin{array}{c}\text { Physical } \\
\text { Variable }\end{array}$ & $\begin{array}{c}\text { Top- } \\
\text { Heaviness }\end{array}$ & $\begin{array}{c}\text { Orien } \\
\text { tation }\end{array}$ & $\begin{array}{c}\text { Factor Name } \\
\text { Curved } \\
\text { Concovity }\end{array}$ & $\begin{array}{c}\text { Angular } \\
\text { Asymmetry }\end{array}$ & $\begin{array}{r}\text { Angular } \\
\text { Concovity }\end{array}$ & $\begin{array}{r}\text { Height } \\
\text { Width }\end{array}$ \\
\hline A. & -13 & 05 & -02 & -09 & -11 & -06 \\
B. & 09 & 05 & -07 & -07 & -07 & -09 \\
C. & 04 & 68 & -13 & -15 & 08 & -06 \\
D. & -05 & 05 & 09 & 04 & -09 & -11 \\
E. & 02 & 04 & 12 & 02 & 05 & 11 \\
F. & -05 & -19 & -02 & -12 & 12 & 51 \\
G. & 53 & 05 & -19 & -01 & -01 & -05 \\
H. & 04 & -01 & 13 & -02 & 50 & 01 \\
I. & -17 & -08 & 53 & 10 & 00 & -02 \\
J. & 00 & 01 & 19 & 55 & -12 & 07 \\
$0 \%$ & 15 & 15 & 14 & 13 & 12 & 12 \\
N & 7 & 3 & 4 & 3 & 4 & 3 \\
\hline
\end{tabular}


Table 10. Pattem of Intersection Factors of 11 Ss Showing Percentages ${ }^{a}$ of Variance of Intersection Accounted for by the Factor Best Described by the Physical Variable Shown on the Left

\begin{tabular}{|c|c|c|c|c|c|c|c|c|c|c|c|}
\hline \multirow{2}{*}{$\begin{array}{l}\text { Physical } \\
\text { Variable }\end{array}$} & \multicolumn{11}{|c|}{ Subject No. } \\
\hline & 1. & 2. & 3. & 4. & 5. & 6. & 7. & 8. & 9. & 10. & 11. \\
\hline A. & 22 & & 20 & 21 & 21 & 20 & 19 & 14 & 20 & 20 & 19 \\
\hline B. & 15 & 19 & 19 & 16 & 18 & 16 & 17 & 13 & & 17 & 17 \\
\hline C. & & & & & 15 & & 17 & 16 & & & \\
\hline D. & 22 & 20 & 19 & 22 & 22 & 21 & 19 & 21 & 20 & 24 & 19 \\
\hline E. & 13 & 15 & 17 & 22 & 16 & 20 & 14 & 19 & 15 & 13 & 19 \\
\hline F. & 12 & 16 & & & 08 & & & & & & \\
\hline G. & & 20 & 12 & 11 & & & 15 & 18 & 14 & & 16 \\
\hline $\mathrm{H}$. & & & & 10 & & 10 & & & 17 & 14 & \\
\hline I. & 16 & & 14 & & & 13 & & & 14 & & \\
\hline J. & & 11 & & & & & & & & 13 & 09 \\
\hline
\end{tabular}

a Percentages may not add to 100 because of rounding.

parameter to enable the user to select any one of an unlimited number of metrics, and an interesting project for future research would be to vary this parameter and see which metric provided the best fit to the input data.

The whole question of metric for similarity judgments of shape probably has its origin in a single paper by Attneave (1950). Attneave scaled the similarity ratings of pairs of simple form stimuli. He found that the scale values for similarity were inconsistent with the simple representation of the stimuli as points in a two-dimensional space describing the only geometric characteristics on which the stimuli differed. He partially resolved this inconsistency by adding a constant to all the psychological scale values. Another problem then arose. The transformed scale values did not conform to a Euclidean distance metric in two dimensions. Instead, they seemed to conform more to a distance metric that Attneave termed the "city-block" model, in which the distance between two points is the sum of the absolute distances between the projections of the points on all reference axes, rather than the length of a straight line connecting the two points.

Torgerson $(1958$, p. 254) treats this topic in some detail, and Hake (1966) presents an excellent critique of Attneave's studies of similarity. Hake discusses the many difficulties in Attneave's methodology and assumptions, and then concludes, "... the city-block model has no real support here. This being so, why has the city-block model, which is based almost entirely on Attneave's data, persisted as a real alternative? A partial answer is that the Attneave data have not been fully explored to see whether they satisfied the critical assumptions involved. A more important answer, probably, is that the city-block model has a strong intuitive appeal."

Hake's exploration of the Attneave data indeed suggests that there is no reason aside from intuitive appeal to use a city-block metric rather than any other metric. But because of its intuitive appeal and because of the serious consideration of this altermative by psychologists, the similarity rating data from the present data were reanalyzed by the Kruskal method using the city-block metric rather than the Euclidean metric. The psychological data from this analysis, then, are in a non-Euclidean space, but the physical data on the shapes are in a Euclidean space. Therefore, the psychophysical analysis of these data involves some thorny mathematical problems which are presently being investigated. The data are not presented here because of these analytic problems and because they would detract from the results already presented. It may be said, however, that the results of the city-block analysis compared to the Euclidean analysis do not show as much consistency among Ss with regard to the number of psychological dimensions required to achieve a good fit to the similarity data. Also, it appears that more dimensions are required in the city-block analysis than in the Euclidean analysis for the average $S$. The goodness-of-fit measure, Stress, reached the 5\% level in from 5-8 dimensions for different Ss using the city-block metric. This finding makes the city-block analysis somewhat suspect at the outset, because a good fit becomes more of a mathematical necessity as the number of dimensions is increased.

\section{Statistical Analysis}

There are two sources of statistical error in the present data analysis. The first source is in the representation of fallible data in the conceptual space through the use of the MDSCAL program. The nature of this source of error can be demonstrated with a hypothetical example. Suppose that a $S$ in the present experiment were in fact making use of five physical dimensions of the stimuli to assess the similarities among all pairs of stimuli. If the $S$ were perfectly consistent in his sorting behavior then the MDSCAL analysis should construct a stimulus configuration such that Stress would be very close to zero when five dimensions were used in the program (assuming that a Euclidean analysis is appropriate). If, however, the $S$ is somewhat inconsistent in his sorting, sometimes he will place a 
stimulus pair in a pile in which it does not belong, and/or he will give different weights to the same physical dimension as he progresses through the sorting task. These inconsistencies will be reflected in the MDSCAL analysis by the appearance of "extra" dimensions. That is, more than five dimensions may be required to achieve a low Stress, the excess being due to statistical error in the data, which the program tries to account for. Notice that in Table 2 the Stress for all Ss when four dimensions are used is in the range that is termed a "fair" fit using Kruskal's verbal description of Stress. It is entirely possible that a fair fit is all that can be achieved using data collected by the present method. If that is the case, then the fifth and sixth dimensions can be said to have no systematic relationship to the perception of shape and should not be used in further analysis. Unfortunately there is no clearcut method by which one can make this determination. As a result, six dimensions were used in the present analysis with the rationale that if one or two of these dimensions were primarily due to errors in the data, then they should show no relationship to physical characteristics of the shapes.

The relationship of the psychological dimensions to the physical dimensions is the second possible source of statistical error. Suppose that one of the psychological dimensions is due primarily to sorting errors made by the $S$. This dimension may show some relationship to the physical dimensions of the stimuli because of a capitalization on chance in the canonical analysis. Here we again are hampered by the lack of an adequate methodology. The only statistical tests available to test the significance of canonical correlation coefficients require the assumption of a multidimensional normal density function (Kendall, 1957). The robustness of these tests under conditions of nonnormality is unknown. We do know, however, that the common univariate statistical tests requiring an assumption of normality may react in bizarre ways to violations of the normality assumption (Bradley, 1964). It is too much to hope that deviations from normality in a multidimensional situation would not produce similar extreme unpredictability. We therefore must rely on logical and psychological analyses of the canonical relationships rather than statistical tests.

If the canonical coefficients in Table 3 are examined for sudden changes in trend as successive coefficients are examined one finds no clear evidence of a discontinuity that would indicate that the smaller coefficients should be regarded as due to chance. An alternative attack on the problem is to require that the smallest canonical correlation to be considered meaningful be greater than .30 , indicating that more than $10 \%$ of the total variance is accounted for by the corresponding canonical vector pair. This is analogous to the tradition in factor analysis of disregarding factor loadings that are not greater than .30. Table 3 shows that the median canonical coefficient for the sixth pair of vectors is .47 and that the smallest coefficient for any $\mathrm{S}$ is .35. Thus, this criterion also gives no evidence for ignoring the canonical vectors with the smallest coefficients.

With no reason to reject any of the six canonical relationships as statistical artifacts, the analysis proceeded as if all dimensions were meaningful, and the IFACs in Tables 5-9 were found. At this point the data again can be examined for possible statistical artifacts. If an individual rotated factor does not account for a substantial proportion of the intersection variance, and if the individual differences with regard to the importance of such a factor are great, then we have reason to suspect the validity of this factor. The implied psychophysical relationship may be considered as due to chance, or, at the least, a real, but relatively unimportant relationship. This view of the data is discussed in the next two sections.

\section{Individual Differences}

The pattern of individual results as summarized in Table 10 indicates that if one considers all the relationships implied by Table 10 as statistically significant then only two of the 11 Ss had similar results. These are Ss Nos. 7 and 8 . This view of the data would indicate that there were 10 subject "types" among the 11 Ss. A more conservative approach would be to ignore those physical variables that account for $10 \%$ or less of the variance in the intersection of the psychological and physical spaces and then compare Ss. Table 10 shows that for Ss Nos. $4,5,6$, and 11 there was one such physical variable for each S. Physical variable $H$ accounted for $10 \%$ of the intersection for Ss Nos. 4 and 7, physical variable $F$ accounted for $8 \%$ of the intersection for $\mathrm{S}$ No. 5, and physical variable $\mathrm{J}$ accounted for $9 \%$ of the intersection for $S$ No. 11. Ignoring these percentages as nonsignificant, we find that Ss 7 and 8 still have similar results and, in addition, Ss 4 and 11 now also show similar results. This view of the data would indicate that there are nine subject types.

A still more conservative approach is to demand that a physical variable account for more than $10 \%$ of the total variance in the psychological space before being considered meaningful. The intersection of the psychological and physical spaces accounted for about $2 / 3$ of the total variance in the psychological space for each of the Ss (see Table 3). Thus, a physical variable that accounts for $15 \%$ of the intersection accounts for about $10 \%$ of the total variance in the psychological space. If in Table 10 we ignore those physical variables accounting for $15 \%$ or less of the intersection, then Ss 3,4,5, and 6 have similar results, and there are eight subject types.

The question of the existence of different per- 
ceptual types among $S$ s with regard to similarity judgments was raised and an analysis was proposed by Tucker and Messick (1963). For this method of analysis one computes a matrix of correlations between all pairs of Ss by summing cross-products of similarity ratings across all pairs of rated objects. This "Subjects-by-Subjects" matrix is factored, and the factors are rotated to simple-structure. Each rotated factor is considered to represent the "viewpoint" of an "ideal subject." The factor loadings that an individual $S$ has on each viewpoint factor represent the degree to which his behavior conformed to that viewpoint throughout the experiment. Homogeneous viewpoint subgroups may be formed on the basis of such an analysis, and the similarity data then may be analyzed within each subgroup.

The Tucker and Messick (1963) type of analysis could have been performed on the data in the present study before the MDSCAL and canonical analyses were performed. However, this was not done because of certain interpretive problems raised in a paper by Ross (1966), in which he shows "... that to combine readings from 'points of view' configurations as assumed in Tucker and Messick's model is not to combine configurations in any simple way." Ross shows in particular that it is possible to generate interpoint distances from the Tucker and Messick method such that no vector space can be found to represent them. These problems were avoided in the present analysis by analyzing each S's data separately.

Silver, Landes, and Messick (1966) report the results of a study in which they used the Tucker and Messick method of analysis on the simflarity judgments of pairs of random shapes. Using $50 \mathrm{Ss}$ they found five viewpoint dimensions. Each of two viewpoints was best described by the data from two separate groups of $14 \mathrm{Ss}$ each, and the remaining three were primarily determined by the data from separate groups of 10,7 , and $5 \mathrm{Ss}$, respectively. A conservative view of the results of the present study indicates eight subject types among the $11 \mathrm{Ss}$, as described earlier. However, the difference between the number of types found in the Silver et al study and the present study is of no great concern because of the differences in methodology and the associated assumptions. The point to be made from these two sets of results is that there are apparently a fair number of different strategies employed by Ss when they are asked to make similarity ratings. Thus, it is possible that studies of similarity in which data are averaged over all Ss produce results representative of no individual $S$.

However, it is interesting to note that if one confines his interest to only four IFACs, Complexity, Curvature, Curvature Dispersion, and Straight-length Dispersion, all Ss seem to be more or less of a single type. They all make use of these physical dimensions of the stimuli, and these four dimen- sions account for the majority of the variance in the perceptual space for most Ss. Thus, with regard to these major variables, there are not wide individual differences.

\section{Psychophysical Dimensions}

Thus far in the discussion of the simple-structure factors that span the psychophysical intersection, I have been careful not to imply that these factors are isomorphic with the characterization of stimuli in the perceptual system. Certainly these physical factors must bear some relation to perceptual processes, or there would be no empirical relationship between similarity judgments and the physical factors. But any rotation of the physical factors would account for exactly the same proportion of the intersection variance, and the "meaning" of the factors would change with each rotation. This problem will be considered in this section.

First, consider the two IFACs that appear in each S's results, the Complexity and Straight-length Disperson factors. Complexity accounts for about $1 / 5$ of the intersection variance, and Straight-length Dispersion accounts for about $1 / 6$ of the intersection variance as shown in Tables 5 and 8 . A comparison of these results with the results of a study by Stilson (1966) is relevant to the meaningfulness of these IFACs.

Stilson had a group of Ss learn nonsense names for a set of triangles of equal area, but of a varying triangular shape. After five learning trials the Ss were asked to recall the proper name for each triangle. Confusion of the names for any given pair of triangles was then used to index the similarity between the two triangles. Four measures of physical similarity between any given pair of triangles were taken by computing the absolute difference between the two triangles on each of four physical measures. The correlation between psychological similarity and physical similarity was then computed for each of the physical measures. Stilson found that the perimeter squared to area ratio ( $\left.\mathrm{P}^{2} / \mathrm{A}\right)$ accounted for about $43 \%$ of the variance among psychological similarities, and a measure of the variability of the side lengths within a triangle accounted for about $40 \%$ of the variance among psychological similarities.

One of the best descriptors of Complexity in the present study is $\mathrm{P}^{2} / \mathrm{A}$, and one of the best descriptors of Straight-length Dispersion is the variance of the lengths of the straight sides of a form as shown in Table 1. Thus, using two widely different sets of stimuli, different measures of similarity, and different analytic techniques, Stilson's study and the present one show essentially the same results: Complexity and Straight-length Dispersion are important predictors of the psychological similarity among forms. This replicability under widely dif- 
ferent conditions supports a view that these physical variables are in some sense basic to information processing in the human perceptual system. It is also encouraging to note that the other two important dimensions highly related to similarity for 10 of the $11 \mathrm{Ss}$ in the present experiment could not have entered into Stilson's study. These dimensions are Curvature and Curvature Dispersion, each of which accounted for slightly less than $1 / 5$ of the intersection variance as shown in Tables 6 and 7 . Stilson's forms were all triangles so that curvature measures were not relevant.

Another study supports the interpretation of Complexity as an analogue of a perceptual process. Stenson (1966) found that the rated complexity of random shapes and the physical factor that is called Complexity here were highly correlated for each of $11 \mathrm{Ss}$, and that no other physical factor contributed significantly to the prediction of complexity ratings. Thus, this physical factor represents a characteristic of form for which a common verbal label exists, and Ss appear to agree on the use of this label. It is reasonable to assume that if we have a common verbal label for a unidimensional, physical characteristic of form, that characteristic is probably representative of processing details in the perceptual system.

Finally, a series of neurophysiological studies, beginning with the work of Hubel and Weisel (1962), is relevant to the interpretation of the intersection factors from the present study. Using single cell recording techniques, these studies indicate that the first stage of information processing in the visual cortex of cats and monkeys consists of activation of cortical cells sensitive only to the presence of lines or edges in an appropriate retinal area. The second stage consists of activation of cells sensitive to change in direction or endings of lines and edges. Thus, the presence of angles or the endings of lines in an appropriate retinal area will cause such a cell to fire. Note in Table 1 that Complexity is defined by the number of turns in the perimeter of a form (or, alternatively, the number of sides) and by the variance of the internal angles in the perimeter. Straight-length Dispersion is defined by measures involving the number of straight sides and their lengths. Thus, these two factors are measures of characteristics of the forms that are directly related to known operating characteristics of cells in the visual cortex.

Curvature and Curvature Dispersion are the two other IFACs that are important for 10 of $11 \mathrm{Ss}$ as shown in Tables 6 and 7 . Subject 2 has no entry in Table 6 and $S$ No. 9 has no entry in Table 7. This is due to the method used to sort the IFACs into homogeneous groups. Subject 2 had an IFAC on which Curvature had a loading of -.47 , but it was not entered into Table 6 because Topheaviness had a loading of .72 on this same IFAC. Therefore, this IFAC was called Topheaviness for S No. 2. However, the loading of -.47 indicates that Curvature probably was involved in this S's similarity judgments. Likewise, $S$ No. 9 had a loading of -.44 for Curvature Dispersion and .48 for Topheaviness on the same IFAC. Thus, this IFAC was called Topheaviness by the sorting criterion used to compile Tables 5-9. Thus, it is probable that Curvature Dispersion was related to this S's similarity ratings also. We may say, then, that all Ss had IFACs corresponding to Curvature and Curvature Dispersion, with each of two Ss having some ambiguity in the IFACs corresponding to one of these physical variables.

There is not as much reason to defend Curvature and Curvature Dispersion as being directly relevant to perceptual processes as there is for the Complexity and Straight-length Dispersion factors discussed above. However, we may note that Curvature Dispersion is the analogue of Straight-length Dispersion and that Curvature apportions a form as to the amount of curvature versus the amount of straightness in the perimeter. Thus, there is a symmetry in this set of four IFACs. One may think of the Curvature and Complexity factors as relatively gross characterizations of a form as a whole, with the former providing a breakdown of type of perimeter and the latter summarizing the configuration of the perimeter. Curvature Dispersion and Straightlength Dispersion provide a more fine-grained analysis of the two types of perimeter discriminated between by Curvature.

All but one of the remaining IFACs may be regarded either as arising from statistical error, or as real, but unstable, physical correlates of perception. Table 9 shows that Topheaviness appears as an IFAC for seven of the $11 \mathrm{Ss}$, while the remaining IFACs appear for only three or four Ss each. Thus, Topheaviness might be considered as a predictor of similarity judgments for the majority of Ss. The combination of the low proportion of variance accounted for and the variability among Ss for the remaining IFACs makes them suspect from a statistical point of view. If one considers them as real effects they indicate that individual differences exist among the judgment systems of the Ss, but little else can be said because of the low probability that any given S's judgments will be related to these IFACs.

\section{Conclusions}

The major conclusion of this research is that while there appear to be minor differences among Ss with regard to the judgment of similarity among forms, four major dimensions appear in each S's data. The simplest physical descriptors of these dimensions are: (1) the proportion of the perimeter of a form that is curved; (2) the complexity of the 
form as indexed by the number of turns in the perimeter and the perimeter-squared area ratio; (3) the variance of the distribution of straight lengths in the perimeter; and (4) the variance of the distribution of curved lengths in the perimeter. Another dimension that appeared for seven of 11 Ss can be described as Topheaviness, as indexed by the skewness of the form in a vertical direction when the form is viewed as a statistical distribution. One may speculate that another dimension that might be important for a different set of forms than those used here would be the horizontal counterpart of Topheaviness; that is, the skewness of a form along a horizontal axis.

It was concluded that the four major physical variables listed above might be more than just statistical correlates of the judgment process: They may bear a resemblance to analytic processes that take place in the perceptual system. This conclusion is based on three considerations: (1) these physical variables form a kind of hierarchical system of analysis; (2) two of these variables are the same as those found to be important in another, quite different study of figural similarity; and (3) recent neurophysiological studies of the visual system have shown that there are cortical cells whose activity is closely linked to aspects of the visual environment similar in nature to two of these four physical variables.

\section{References}

Amoult, M. D. Prediction of perceptual responses from structural characteristics of the stimulus. Percept. mot. Skills, 1960, 11, 261-268.

Attneave, F. Physical determinants of the judged complexity of shapes. J. exp. Psychol., 1957, 53, 221-227.

Attneave, F. Dimensions of similarity. Amet. J. Psychol., 1950, $63,516-556$.

Attneave, F., \& Amoult, M. D. The quantitative study of shape and pattern perception. Psychol. Bull., 1956, 53, 252-271.

Bennett, J. F. Determination of the number of independent parameters of a score matrix from the examination of rank orders. Psychometrika, 1956, 21, 383-393.

Bennett, J. F., \& Hays, W. L. Multidimensional unfolding: determining the dimensionality of ranked preference data. Psychometrika, 1960, 25, 27-44.

Bradley, J. V. Studies in research methodology: VI. The central limit effect for a variety of populations and the robustness of $Z$, t, and F. AMRL Technical Report 64-123, Aerospace Medical Research Laboratories, Wright-Patterson Air Force Base, Ohio, December, 1964.

Hake, H. W. The study of perception in the light of multivariate methods. In R. B. Cattell (Ed.), Handbook of multivariate experimental psychology. Chicago: Rand-McNally, 1966. Pp. 502-534.

Hotelling, H. Relations between two sets of variates. Biometrika, $1936,28,321-377$.

Hotelling, H. The most predictable criterion. J. educ. Psychol, $1935,26,139-142$.

Hubel, D. H., \& Wiesel, T. N. Receptive fields, binocular interaction, and functional architecture in the cat's visual cortex. J. Physiol., 1962, 160, 106-154.

Kendall, M. G. A course in multivariate analysis. London: Charles Griffin \& Co., Ltd., 1957.

Kruskal, J. B. Multidimensional scaling by optimizing goodness of fit to a nonmetric hypothesis. Psychometrika, 1964, 29 1-27 and $115-129$.

Richardson, R. W. Multidimensional psychophysics. Psychol. Bull., 1938, 35, 659-660.

Ross, J. A remark on Tucker and Messick's "Points of view" analysis. Psychometrika, 1966, 31, 27-31.

Shepard, R. N. The analysis of proximities: multidimensional scaling with an unknown distance function. I, II. Psychometrika, $1962,27,125-139$ and 219-245.

Silver, C. A., Landes, D., \& Messick, S. Multidimensional analysis of visual form: an analysis of individual differences. Amer. J. Psychol., 1966, 79, 62-72.

Stenson, H. H. The physical factor structure of random forms and their judged complexity. Percept. \& Psychophys., 1966, 1, 303-310.

Stilson, D. W. A psychophysical investigation of triangular form. Amer. J. Psychol, 1966, 79, 258-264.

Torgerson, W. S. Theory and methods of scaling. New York: Wiley, 1958.

Tucker, L、 R., \& Messick, S. An individual differences model for multidimensional scaling. Psychometrika, 1963, 28, 333-367. Young, G., \& Householder, A. S. Discussion of a set of points in terms of their mutual distances. Psychometrika, 1938, 3, 19-22.

\section{Notes}

1. The research reported in this paper was sponsored by the Aerospace Medical Research Laboratory, Aerospace Medical Division, Air Force Systems Command, Wright-Patterson Air Force Base, Ohio, under contract number F 33615-67-C-1280 with Antioch College, Yellow Springs, Ohio. This paper has been identified by the Aerospace Medical Research Laboratory as AMRL-TR-67-131. Further reproduction is authorized to satisfy needs of the United States Government.

2. The author wishes to thank Dr. J. B. Kruskal of Bell Telephone Laboratories for providing the computer program necessary for one phase of this study.

(Accepted for publication Norember 4, 1967.)

\section{Mathematical Appendix}

The canonical analysis of the relationship between the psychological space from the MDSCAL analysis and the physical space describing forms yields two weight matrices, $W$ and $V$, for each subject, such that:

\section{and}

(1) $W^{\prime} \Phi^{\prime} \dot{\Psi} V=C$,

(2) $W^{\prime} \Phi^{\prime} \Phi W=V^{\prime} \Psi^{\prime} \Psi V=I$,

where $\Phi$ is a matrix in which each column shows the scores of the forms on a simple-structure physical factor, and $\Psi$ is a matrix in which each column shows the scores of the forms on a psychological reference axis. Each column in both matrices has a zero mean. $C$ is a diagonal matrix of canonical correlation coefficients, and $I$ is the identity matrix.

Because $\Phi$ is a factor score matrix, $\Phi^{\prime} \Phi=I$, and from (2) it can be seen, then, that $W^{\prime} W=I$. Note, however, that $W^{\prime} W^{\prime} \neq I$ because $W$ is not square for the data in the present study. (Its order is 10x6).

If both sides of (1) are multiplied by $\mathrm{C}^{-1}$, then we have:

(3) $\left(C^{-1} W^{\prime}\right) \Phi^{\prime} \Psi V=I$. 
The only matrix that will yield the identity when premultiplied by $\mathrm{C}^{-1} \mathrm{~W}^{\prime}$ is WC. Therefore:

(4) $\Phi^{\prime} \Psi V=W C$.

The left side of (4) shows that WC is a matrix showing the projections of the original physical variables (factors) onto the canonical vectors of the psychological space. These canonical vectors may be transformed by an orthogonal matrix, $T$, such that $\Phi^{\prime} \Psi$ V T has simple-structure. This is equivalent to transforming WC so that WCT has simple-structure. Then

$$
\text { (5) } \begin{aligned}
\operatorname{tr}\left((W C T)^{\prime}(W C T)\right) & =\operatorname{tr}\left(C^{2}\right) \\
& =\text { Intersection Variance. }
\end{aligned}
$$

Thus the columns of WCT were called Intersection Factors. 